\title{
Examining the reliability and factor structure of the Malay version of the Recovery Self-Assessment in a sample of individuals with schizophrenia
}

\begin{abstract}
Objective: Without a reliable and valid tool, it is neither possible to gauge recovery orientation of persons with serious mental illness nor to evaluate whether existing mental health system encourages recovery in both its policies and current practice in Malaysia. Therefore, the aim of this study was to examine the reliability and factor structure of the Malay version of the Recovery Self-Assessment (RSA-PIRV-M) in a psychiatric sample. Method: Psychiatric patients were eligible for recruitment to the study, when they fulfilled the following criteria: a diagnosis of schizophrenia, were aged between 18- and 65-years-old, and were able to give consent themselves. We recruited 118 individuals with schizophrenia. They were selected via systematic random sampling technique. All RSA-PIRV-M items were derived from the parent scale. These items were translated based on established procedures. The reliability estimates of the RSA-PIRV-M were based on Cronbach's alpha. We performed confirmatory factor analyses to examine the factor structure of the RSA-PIRV-M. Results: All Cronbach's alphas for the RSA-PIRV-M subscales were at least .70. With respect to the factor structure of the RSA-PIRV-M, our structural equation modeling findings suggest a five-factor model encompassing life goals, involvement, diversity of treatment options, choice, and individually-tailored services. Conclusions and implications for practice: The interpretation of our findings is limited by small sample size, unique sample characteristics, and lack of further evidence for convergent validity. Notwithstanding these limitations, the RSA-PIRV-M is a promising tool in bridging gaps between our knowledge on recovery orientation and existing mental health service provision in Malaysia.
\end{abstract}

Keyword: Recovery Self-Assessment; Schizophrenia; Mental illness; Mental health 